\title{
Evaluating the Cost of Line Capacity Limitations in Aggregations of Commercial Buildings
}

Ziras, Charalampos; Delikaraoglou, Stefanos; Kazempour, Jalal; You, Shi; Bindner, Henrik W.

Published in:

Proceedings of the 52nd International Universities' Power Engineering Conference

Link to article, DOI:

10.1109/UPEC.2017.8231895

Publication date:

2017

Document Version

Peer reviewed version

Link back to DTU Orbit

Citation (APA):

Ziras, C., Delikaraoglou, S., Kazempour, J., You, S., \& Bindner, H. W. (2017). Evaluating the Cost of Line Capacity Limitations in Aggregations of Commercial Buildings. In Proceedings of the 52nd International Universities' Power Engineering Conference IEEE. https://doi.org/10.1109/UPEC.2017.8231895

\section{General rights}

Copyright and moral rights for the publications made accessible in the public portal are retained by the authors and/or other copyright owners and it is a condition of accessing publications that users recognise and abide by the legal requirements associated with these rights.

- Users may download and print one copy of any publication from the public portal for the purpose of private study or research.

- You may not further distribute the material or use it for any profit-making activity or commercial gain

- You may freely distribute the URL identifying the publication in the public portal 


\title{
Evaluating the Cost of Line Capacity Limitations in Aggregations of Commercial Buildings
}

\author{
Charalampos Ziras, Stefanos Delikaraoglou, Jalal Kazempour, Shi You, Henrik W. Bindner \\ Department of Electrical Engineering \\ Technical University of Denmark \\ Email: $\{$ chazi, stde, seykaz, sy, hwbi\}@elektro.dtu.dk
}

\begin{abstract}
The trend towards electrification of the heating sector in many cases leads to the replacement of fossil-fueled heating systems with electric heat pumps. This may result to significantly higher consumption and potentially violations of the distribution grid operational limits. We propose a day-ahead optimization strategy to assess the cost of imposing capacity limitations in the total consumption of individual buildings, as well as aggregations of buildings. We show that such capacity limitations lead to an increase for the buildings operational costs, which can be interpreted as the value of these limitations. Based on such calculations, the aggregator can value capacity-limitation services to the distribution system operator. Moreover, the value of aggregation is also highlighted, since it leads to lower costs than imposing the same total capacity limitation on individual buildings.
\end{abstract}

Keywords- Commercial buildings aggregation, demand response, DSO services, line capacity limit.

\section{INTRODUCTION}

The electrification of the heating sector poses a number of challenges to the operation of power systems. One of these challenges is the increased loads in the low voltage grids by resources with large consumptions, such as electric heat pumps (HPs) or electric vehicles (EVs). Moreover, the potential profits from the aggregation of such resources and the participation in the power markets via aggregators is gradually being realized. These aggregators are responsible for controlling such loads and represent them towards the system operator and the power markets. A topic of significant undergoing research is the impact of these loads to the distribution grids [1]. Traditionally, distribution grids have been designed with large operating margins and in most cases line congestions are rare, but are expected to occur more frequently when significant numbers of HPs or EVs are installed and controlled via aggregators.

There is a large variety of approaches for handling congestions and voltage problems in the distribution grids, such as time-of-use tariffs [2], distribution locational marginal pricing [3] and contracted capacity limits [4]. In this study we focus on the last approach, which is a relatively mature method, easy to implement and with limited requirements in terms of monitoring and communication. A number of distribution

The authors would like to acknowledge the financial support of the EUDP project, Ecogrid 2.0, No 64015-0082.

978-1-5386-2344-2/17/\$31.00 (C) 2017 IEEE system operators (DSOs) charge their customers based on a contracted capacity, so that they can have some guarantees on the maximum consumption. Moreover, the increased shares of controllable distributed energy resources (DERs) in the distribution grids may lead the DSOs to trade DSO services with the aggregators to ensure the safe grid operation and avoid expensive upgrades. It is therefore useful for aggregators, who manage large loads on behalf of their customers, to have a method to calculate the optimal contracted capacity or the value of DSO services based on load limitations, which in the general case can be time-varying and involve both a minimum and a maximum consumption.

Physical line capacity limits (to the point of connection with the grid) can be also exceeded in buildings where substantial new large loads are installed, such as EV charging stations and large HPs. In this paper we focus on a realistic case, where the gas-boiler based heating system of a commercial building is supplemented with HPs. We propose an optimization strategy in order to quantify the cost of a capacity limitation on individual and aggregated buildings. This limit can be either physical (the total load exceeding the actual line capacity under certain conditions) or artificial and imposed by the DSO. In the first case, the aggregator can use this method to evaluate whether it is more economical to operate under a reduced flexibility or to upgrade the connection. In the second case, the aggregator can calculate the cost of an imposed capacity limitation and pay or trade the optimal capacity.

Our work is inspired by [5], where a capacity-controlled demand side management paradigm is proposed and each customer is assigned a capacity limit by the aggregator, which the customer is responsible to respect. The authors do not focus on optimizing the day-ahead (DA) schedule, but rather construct a demand curve for the aggregated capacity limit against a shadow price, which corresponds to the discomfort cost of curtailing load. The aggregator uses this curve to bid in the DA market and minimize the cost of purchasing the required energy for its customers.

In our case we propose an optimal bidding strategy in the DA market, considering the uncontrolled load uncertainty, i.e. the consumption excluding heating which cannot be controlled or curtailed. The use of on-off HPs, which must switch off in case of large uncontrolled load values, significantly changes the problem. We formulate it as a mixed integer stochastic problem (MISP) with recourse decisions, to obtain an optimal day-ahead schedule against load uncertainty. We then calculate 
the average expected costs under various DA prices and varying capacity limitations, to value the cost incurred by the reduced flexibility.

The remainder of the paper is organized as follows. In Section II we introduce the building model and the deterministic mixed-integer optimization problem to minimize costs. In Section III we introduce the uncontrolled load uncertainty and we present the two-stage MISP, whereas in Section IV we present the simulation results considering a varying capacity limit for a single building. In Section $\mathrm{V}$ we present results for an aggregation of 3 buildings and we show the benefit of aggregated capacity limits compared to imposing them on individual buildings. Finally, in Section VI we discuss the applicability of the proposed method and the direction of our future work.

\section{DETERMINSTIC BUILDING SCHEDULING}

In this section we introduce the building model used in our study, as well as its deterministic DA scheduling. The focus of this paper is on presenting a methodology to evaluate the cost of line capacity limitations on the operation of buildings, and therefore a simple representation of the heat demand and temperature dynamics is used. We assume that the evolution of a building's lumped temperature $T$ at each time step $t$ can be described by

$$
T_{t+1}=a T_{t}+(1-a)\left(T_{\text {out }, t}+Q_{t} R\right),
$$

where $a=e^{-\Delta t /(R C)}, R$ is the thermal resistance, $C$ is the thermal capacitance, $T_{\text {out }}$ is the outside temperature, $\Delta t$ is the discretization time step, and and $Q_{t}$ is the heat provided to the building in $\mathrm{kW}$. We must note here that $\Delta t$ is the discretization time-step duration for the building model and the decision variables, and is in general different than the hourly timesteps of the market; in this study we use $\Delta t=15$ minutes. This differentiation is necessary because the variability of the uncontrolled load and the imposed restrictions on the heat pumps' operation cannot be captured by hourly time steps. We thus introduce $n_{\mathrm{p}}$ (which in our study is equal to 4) as a normalization factor to transform power into energy.

We denote by $u_{k, t}$ the binary state of the $k$-th HP at time step $t$, and by $Q_{t}^{\mathrm{b}}$ the heat provided by the boiler. The total heat provided in the building is calculated by

$$
Q_{t}=\sum_{k=1}^{N_{\mathrm{HP}}} u_{k, t} P_{\mathrm{nom}} \eta+\eta_{b} Q_{t}^{\mathrm{b}}
$$

where $N_{\mathrm{HP}}$ is the number of HPs, $P_{\text {nom }}$ is the nominal capacity of the HPs, and $\eta$ is the coefficient of performance (COP) of the HPs; the boiler capacity is denoted by $Q_{\text {nom }}$ and the efficiency by $\eta_{\mathrm{b}}$. For notation simplicity and without loss of generality we consider buildings equipped with HPs of the same nominal capacity and COP. Temperature must be always kept within the specified limits

$$
T_{\min , t} \leq T_{t} \leq T_{\max , t}
$$

To simplify the analysis and interpretation of the results, we assume that no heat storage tank exists, or that its size is so small that it can be neglected. The total HP and the uncontrolled load $\left(P_{t}\right)$ consumption are limited by the line capacity limit $P_{\text {cap }}$, resulting in the following constraint

$$
\sum_{k=1}^{N_{\mathrm{HP}}} u_{k, t} P_{\mathrm{nom}}+P_{t} \leq P_{\text {cap }}
$$

It must be noted that $P_{t}$ is given as an input in the deterministic case and is always smaller than $P_{\text {cap }}$, since line congestion was impossible prior to the installation of the HPs. The deterministic cost-minimization problem over an optimization horizon of $N_{\mathrm{T}}$ operational time steps and $N$ hours for a single building can be expressed as the following mixed integer problem

$$
\min _{\mathbf{u}, \mathbf{Q}^{\mathrm{b}}}\left(1 / n_{\mathrm{p}}\right) \sum_{j=1}^{N}\left[\sum_{t=m}^{j n_{\mathrm{p}}} Q_{t}^{\mathrm{b}} c_{\mathrm{h}}+\lambda_{j} \sum_{t=m}^{j n_{\mathrm{p}}} \sum_{k=1}^{N_{\mathrm{HP}}} u_{k, t} P_{\text {nom }}\right]
$$

where $m=(j-1) n_{\mathrm{p}}+1$, and $\lambda_{t}$ and $c_{\mathrm{h}}$ express the DA electricity price and the natural gas cost respectively, both in $\mathrm{DKK} / \mathrm{kWh}$. While the double summation over time may seem counter-intuitive, it is required due to the hourly resolution of the DA electricity prices and the imbalance costs. Problem (5) must be solved subject to constraints (1)-(4), as well as

$$
\begin{aligned}
u_{k, t} & \in\{0,1\} \\
0 \leq Q_{t}^{\mathrm{b}} & \leq Q_{\text {nom }} .
\end{aligned}
$$

Notice that the (fixed) terms for the uncontrolled load $P_{t}$ do not appear in the objective function of the deterministic case because they do not affect the solution, whereas the fixed term $1 / n_{\mathrm{p}}$ in the objective function can be omitted.

\section{TWO-STAGE DAY-AHEAD STOCHASTIC OPTIMIZATION OF AN AGGREGATION OF BUILDINGS}

\section{A. Load uncertainty and recourse decisions}

The decision making process of the aggregator and the way load uncertainty is modelled and unveiled defines to a large extent the formulation of the stochastic optimization problem. At first, the aggregator must decide upon the scheduling of the gas boiler and buy the electric energy from the DA market (the stochastic uncontrolled load and the load corresponding to the consumption of the HPs), but at the same time position itself in an optimal manner against the load uncertainty realization.

During operation, depending on the uncontrolled load realization, one or more HPs may be forced to switch off due to the line capacity limit, or can be switched on if the realized uncontrolled load is low enough. Moreover, an energy imbalance between the DA schedule and the actual consumption is penalized by forcing the aggregator to buy/sell the energy deficit/surplus at the regulating-power price. The aggregator (or the buildings, depending upon the control architecture) can take recourse decisions and act on the realized load scenarios. In our case these decisions include the re-scheduling of the HPs and the gas boiler, whereas the DA schedule remains fixed.

We differentiate between the $N$ hourly time steps (24 in our case) for the DA energy schedule and the corresponding 
DA prices, as well as the hourly imbalance costs, and the operational $N_{\mathrm{T}}$ time steps (96 in our case) for the HPs and the gas boiler, as well as the uncontrolled load uncertainty. This differentiation is necessary because the variability of the uncontrolled load, the imposed restrictions on the HPs operation, and the temperature dynamics cannot be captured well by hourly time steps. As a result, the operational time step in this study will be 15 minutes, and the average consumption over 4 steps will be considered for the imbalance cost.

\section{B. Two-stage stochastic optimization formulation}

The aggregator faces a multistage optimization problem, because the uncertainty unfolds at each time-step and the recourse decisions affect the decision variables at the remaining optimization horizon, due to the time-coupling caused by the temperature dynamics and the hourly imbalance-cost granularity.

If the uncertainty is represented by a finite number of scenarios at each time-step, then the resulting multi-stage mixedinteger stochastic optimization problem becomes a particularly challenging program. We can imagine the uncertainty as a decision tree with the time steps of the optimization horizon as nodes; the aggregator observes the uncertainty at time-step $t$, considers the states at the previous time-step $t-1$ and takes actions to minimize the expected future cost by solving an optimization problem for the remaining time steps; this results in a very large nested optimization problem for a 96 steps optimization horizon.

If a relatively large number of buildings is considered, then the resulting problem is very hard to solve, at least in a centralized manner. In [6], an extension of the Benders decomposition algorithm is proposed, in order to decompose and solve such problems. A commonly used method to simplify the problem is to reduce it to a two-stage stochastic integer program cite [7], [8] and derive the DA schedule (purchased energy and gasboiler scheduling) by considering scenarios which represent the uncertainty for the whole optimization horizon.

\section{Problem formulation}

Assuming perfect DA price forecast and no disturbances affecting the buildings' temperatures, the aggregator bids in the DA market with the objective of minimizing its costs under uncertain uncontrolled loads. The aggregator's costs consist of the electricity costs, the natural gas consumption costs, and the imbalance costs, incurred by the mismatch between the total DA scheduled consumption and the actual total load of all the buildings. In this study we assume that the aggregator can buy or sell the energy deviation resulting from its DA schedule at the spot price, but is penalized by a factor proportional to the DA price, as will be discussed later.

The DA decisions are the bidded DA schedule $\mathbf{P}_{\mathbf{D A}}$ expressed in $\mathrm{kW}$, the schedules of the HPs $\mathbf{u}$, and the boilers load $\mathrm{Q}^{\mathrm{b}}$ expressed in thermal $\mathrm{kW}$. For $N_{\mathrm{b}}$ buildings, $N_{\mathrm{b}, i}$ HPs in each building $i, N$ time steps for purchasing energy in DA, and $N_{\mathrm{T}}$ operational time steps, $\mathbf{P}_{\mathbf{D A}} \in \mathbb{R}^{N_{\mathrm{b}} \times N}, \mathbf{u} \in \mathbb{R}^{N_{\mathrm{b}, i} \times N_{\mathrm{b}} \times N_{\mathrm{T}}}$, and $\mathbf{Q}^{\mathrm{b}} \in \mathbb{R}^{N_{\mathrm{b}} \times N_{\mathrm{T}}}$.
Each scenario $\omega$ has a probability of occurrence $\pi_{\omega}$ and corresponds to a set of realized $P_{t, \omega}^{i}$ consumptions for each time-step $t$ at each building $i$. The recourse decisions include the decisions to switch a heat pump on $\left(u_{k, t, \omega}^{i,+}\right.$ for HP $k$ will then become equal to 1$)$ or off $\left(u_{k, t, \omega}^{i,-}\right.$ will become 1$)$ and the re-scheduling of the boilers $\left(Q_{t, \omega}^{\mathrm{b}, i, \mathrm{rc}}\right)$. To simplify notation we introduce the term $P_{\text {act }}(t, i, \omega)$ for the actual consumption of building $i$ at time step $t$ and scenario $\omega$

$$
P_{\text {act }}(t, i, \omega)=\sum_{k=1}^{N_{i}}\left(u_{k, t}^{i}+u_{k, t, \omega}^{i,+}-u_{k, t, \omega}^{i,-}\right) P_{\text {nom }}^{i}+P_{t, \omega}^{i} .
$$

The optimization problem then takes the following form

$$
\begin{aligned}
\min _{\mathbf{W}} & \sum_{j=1}^{N} \sum_{i=1}^{N_{\mathrm{b}}}\left[P_{\mathrm{DA}, j}^{i} \lambda_{j}+\left(1 / n_{\mathrm{p}}\right) \sum_{t=m}^{j n_{\mathrm{p}}} Q_{t}^{\mathrm{b}, i} c_{\mathrm{h}}\right] \\
& +\sum_{\omega \in \Omega} \pi_{\omega}\left[\left(1 / n_{\mathrm{p}}\right) \sum_{t=1}^{N_{\mathrm{T}}} \sum_{i=1}^{N_{\mathrm{b}}} Q_{t, \omega}^{\mathrm{b}, i, \mathrm{rc}} c_{\mathrm{h}}+\sum_{j=1}^{N} \sum_{i=1}^{N_{\mathrm{b}}} B(j, i, \omega)\right] \\
\mathrm{s.t.} \quad & u_{k, t}^{i}, u_{k, t, \omega}^{i,+}, u_{k, t, \omega}^{i,-} \in\{0,1\} \\
& 0 \leq Q_{t}^{\mathrm{b}, i} \leq Q_{\mathrm{nom}}^{i} \\
& P_{\mathrm{act}}(t, i, \omega) \leq P_{\mathrm{cap}}^{i} \\
& u_{k, t}^{i}+u_{k, t, \omega}^{i,+} \leq 1, \quad u_{k, t, \omega}^{i,-} \leq u_{k, t}^{i} \\
& T_{t+1, \omega}^{i}=a^{i} T_{t, \omega}^{i}+\left(1-a^{i}\right)\left(T_{\mathrm{out}, t}^{i}+Q_{t, \omega}^{i} R^{i}\right) \\
& T_{\mathrm{min}, t}^{i} \leq T_{t, \omega}^{i} \leq T_{\mathrm{max}, t}^{i} \\
& Q_{t, \omega}^{i}=\sum_{k=1}^{N_{i}}\left(u_{k, t}^{i}+u_{k, t, \omega}^{i,+}-u_{k, t, \omega}^{i,-}\right) P_{\mathrm{nom}}^{i} \eta^{i} \\
& +\left(Q_{t}^{\mathrm{b}, i}+Q_{t, \omega}^{\mathrm{b}, i, \mathrm{rc}}\right) \eta_{\mathrm{b}}^{i} \\
& Q_{\mathrm{nom}}^{i}-Q_{t}^{\mathrm{b}, i} \geq Q_{t, \omega}^{\mathrm{b}, i, \mathrm{rc}} \geq-Q_{t}^{\mathrm{b}, i}
\end{aligned}
$$

where $\mathbf{W}$ contains all the optimization variables and $\Omega$ is the set of all the scenarios. The indexes for the constraints are omitted for simplicity. The imbalance of hour $j$ is calculated by subtracting the total realized load (averaged over 4 time steps) from the hourly DA schedule and this value is multiplied by a factor $\rho_{j}$, which is a fraction of the DA price. $B(j, i, \omega)$ expresses the scenario-specific cost over each hourly time step $j$ and is given by

$$
\begin{aligned}
B(j, i, \omega) & =\left[P_{\mathrm{DA}, j}^{i}-\left(1 / n_{\mathrm{p}}\right) \sum_{t=m}^{j n_{\mathrm{p}}} P_{\mathrm{act}}(t, i, \omega)\right] \lambda_{j} \\
& +\lambda_{j} \rho_{j}\left|P_{\mathrm{DA}, j}^{i}-\left(1 / n_{\mathrm{p}}\right) \sum_{t=m}^{j n_{\mathrm{p}}} P_{\mathrm{act}}(t, i, \omega)\right|
\end{aligned}
$$

$B$ consists of 2 terms. The first one represents the excess or deficit of the energy consumption, which is sold back or bought to/from the system on the spot price. The second term penalizes such imbalances with $\rho_{j}$ times the spot price. Logical constraints (8e) ensure that a HP which was DA-scheduled 
TABLE I: Building Parameters

\begin{tabular}{c|c|c}
\hline Parameter & Description & Value \\
\hline$\eta_{\mathrm{b}}(-)$ & Boiler efficiency & 0.9 \\
$\eta(-)$ & COP & 3 \\
$\rho(-)$ & Imbalance penalty factor & 0.2 \\
$Q_{\text {nom }}(\mathrm{kW})$ & Boiler capacity & 100 \\
$P_{\text {nom }}(\mathrm{kW})$ & HP capacity & 12 \\
$T_{\min }\left({ }^{\circ} \mathrm{C}\right)$ & Minimum temperature & 21.5 \\
$T_{\max }\left({ }^{\circ} \mathrm{C}\right)$ & Maximum temperature & 22.5 \\
\hline
\end{tabular}

to be on can only be turned off and a HP scheduled to be off can only be turned on. Constraints (8c) and (8i) ensure that the boiler always operates within the allowed limits, (8d) enforces the line capacity limit, and the rest are the perscenario constraints similar of the deterministic problem. We must note at this point that the inclusion of an absolute-value term in (9) results in a non-linear mixed integer problem (NLMIP). However, it is straightforward to reformulate the problem by replacing the absolute value and imposing a set of linear constraints, thus retaining the linear structure of the problem.

\section{TEST CASE FOR A SINGLE BUILDING}

In this section we investigate the effect of a varying line capacity limit in the operating costs of a single building. We used real-consumption data of a Danish school building and fitted the RC parameters to obtain similar heat consumption values. 10 scenarios for the uncontrolled load were used, 3 of which are shown for illustrative purposes in Fig. 1. A relatively large load variability can be observed between $08.00: 18.00$, whereas this variability is considerably lower during the rest of the day. The building is equipped with 2 HPs and we use constant temperature limits. The HP characteristics were taken from [9] and the parameters are presented in Table I.

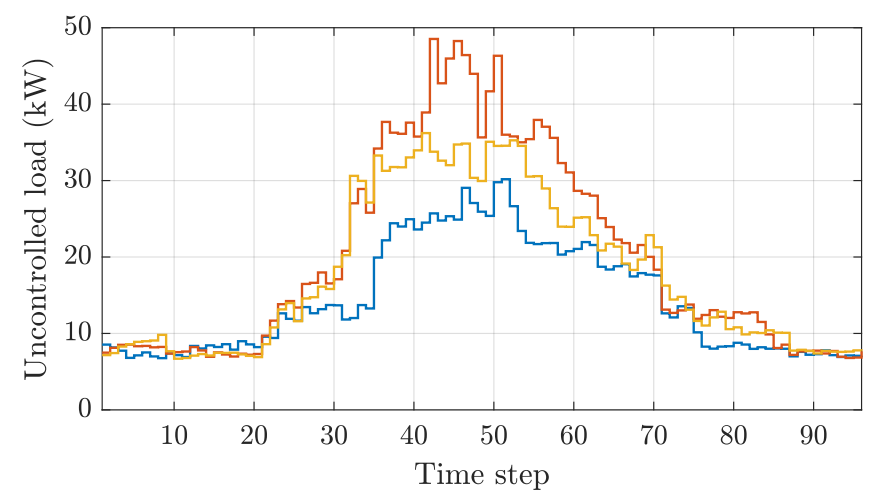

Fig. 1: 3 different scenarios showing the uncontrolled load consumption during a day.

We conducted extensive simulations with typical DA prices for Eastern Denmark [10] and winter temperature patterns, while considering 10 different uncontrolled load scenarios. For our simulations we used the Matlab-YALMIP interface [11] and the Gurobi solver [12]. We observed that the value of the stochastic solution is relatively small; in other words, the resulting cost by solving the MISP for each scenario realization is similar to the one obtained by solving the deterministic MIP, considering the expectation (mean values) of the uncontrolled load from the 10 scenarios.

This is attributed to 2 reasons. First, under perfect heatdemand forecast, the building is able to react to the uncertainty realization with a very small cost. Consider the case where a low uncontrolled load scenario is realized; one or more HPs (originally scheduled as off) can now operate and thus decrease the gas cost incurred by the use of the boiler. Second, imbalance costs are calculated as hourly energy imbalances, which can be compensated better in the course of 4 operational steps.

To illustrate the benefit of solving the MISP, we considered 2 different outside temperature scenarios and modified constraint $(8 \mathrm{~g})$ to make it scenario-dependent. Scenarios $1-10$ refer to the first temperature realization (and 10 different uncontrolled load realizations) and scenarios $11-20$ to the second. In all cases, as seen in Fig. 2, the cost achieved by the stochastic problem is smaller compared to the deterministic (where the expected values of the outside temperature and uncontrolled load are used), due to the smaller imbalance costs.

In reality, the actual operating range for the HPs at time step $t$ is not $P_{\text {cap }}-P_{t, \omega}^{i}$ for a scenario $\omega$. In fact this value is the upper bound of the operating range because within period $t$, the uncontrolled load will take instantaneous values which are at least equal to $P_{t, \omega}^{i}$. Depending on the type of protection, a large peak sustained for more than a specific duration may require a heat pump to switch off, which will remain off for a given period due to the lockout effect. Such lockout durations, prohibit the frequent switching of the compressors to avoid wear of the equipment. This large variability in the sub-minute range is very common and we believe that it may significantly affect the operation of on-off HPs and the heating costs. We intend to investigate the effect of such fast load variations, based on real measurements, in our future work.

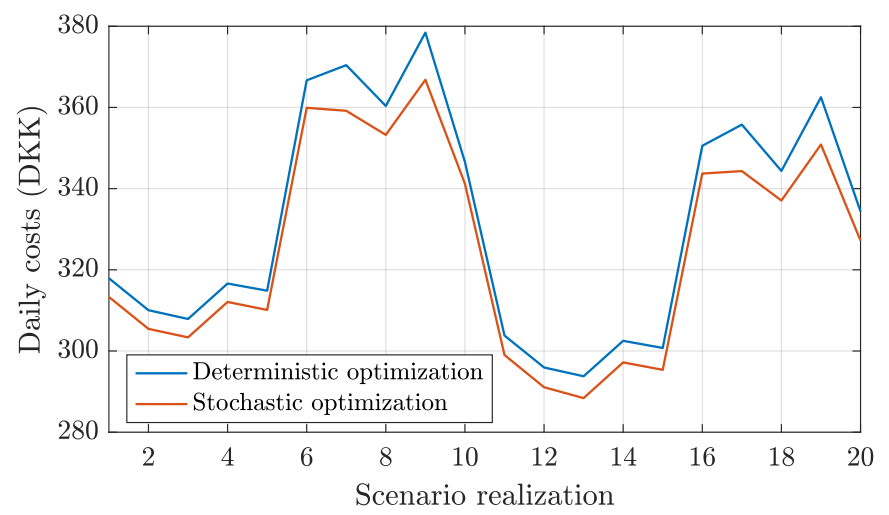

Fig. 2: Daily costs for the deterministic and stochastic problems with $\rho=0.5, c_{\mathrm{h}}=1.5 \mathrm{DKK} / \mathrm{kWh}$ and $P_{\text {cap }}=60 \mathrm{~kW}$.

Next, we focused on the effect $P_{\text {cap }}$ on the average daily costs. We used 20 random daily spot prices profiles and a typ- 
ical winter temperature pattern; we used an hourly imbalance cost as is the current practice. The actual electricity costs are much higher due to the presence of taxes and fees and the existence of various charging schemes for gas and electricity, i.e. different fees per DSO or different taxes depending on the use of electricity, makes the evaluation harder. For this reason we use the spot prices for electricity and 3 scenarios for gas prices (without additional tariffs and taxes).

The resulting average daily costs are shown in Fig. 3, where a cost reduction with the increase of $P_{\text {cap }}$ is observed, as expected. The capacity limit ranges from $50-70 \mathrm{~kW}$, because a limit equal to $50 \mathrm{~kW}$ guarantees that the uncontrolled load consumption is always met without curtailment, whereas the upper limit of $70 \mathrm{~kW}$ is set as the maximum possible total consumption. We can see that the results are very sensitive to the gas price, because a strict line limit leads to the use of the gas boiler when the uncontrolled load is high. It is interesting to note that in all cases a $P_{\text {cap }}>65 \mathrm{~kW}$ offers no additional cost reduction and is essentially free for the aggregator. For smaller limits, the reduced flexibility results in higher expected costs and therefore can be evaluated and sold to the DSO as a capacity limit.

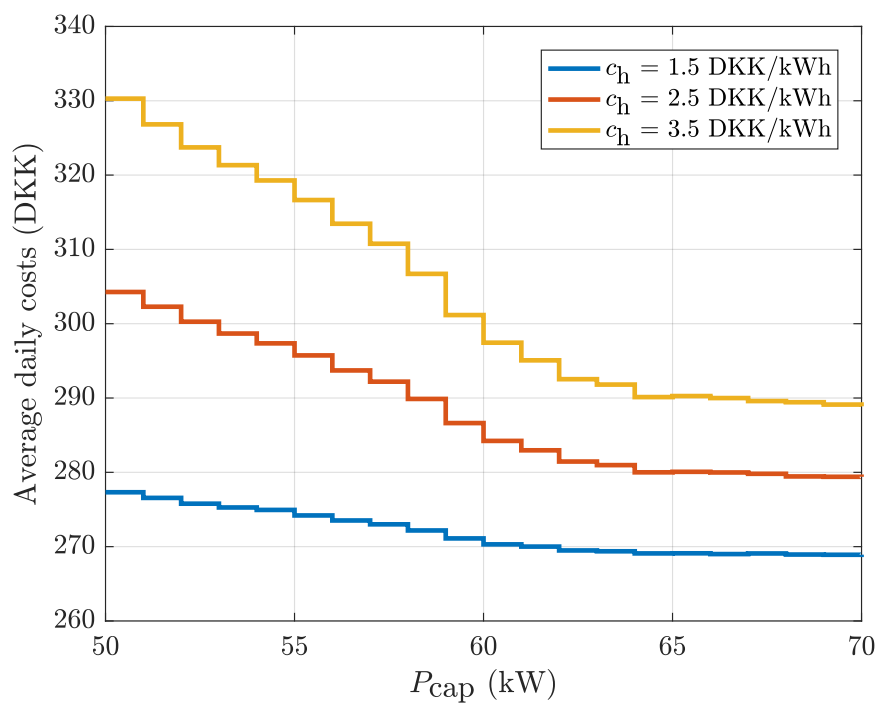

Fig. 3: Average daily costs for a varying capacity limit and for 3 different gas prices.

\section{LINE LIMITS IN AGGREGATIONS OF BUILDINGS}

In this section we investigate the effect of line limitations in aggregations of buildings. To reduce the computational burden we only consider 3 uncontrolled load scenarios with equal occurrence probabilities, and more specifically the ones shown in Fig. 1: a 'low', a 'medium', and a 'high' load scenario. For these simulations we fixed $c_{\mathrm{h}}=2.5 \mathrm{DKK} / \mathrm{kWh}$, hourly imbalances with $\rho=0.2$, and we calculated the average daily costs for 20 different DA price profiles.

We used an aggregation of 3 similar buildings and first we imposed individual capacity limits, ranging from 50 to $70 \mathrm{~kW}$.
Next, we imposed an aggregated capacity limit by modifying constraint (8d) so as to correspond to the total load of the 3 buildings. If we assume no correlation between the realizations of the uncontrolled load scenarios, we can identify 10 distinct scenarios (for identical buildings), out of the 27 combinations. However, the probability $\pi_{\omega}$ of each scenario is not equal to 0.1 , but is calculated by dividing the number of combinations which result to a specific scenario with the total number of combinations.

The resulting costs are shown in Fig. 4, where the average daily costs of the 3 buildings are shown under individual capacity limits and an aggregated capacity limit. Again we observe that the costs decrease as the capacity limit increases, due to the increased flexibility and utilization of the HPs, instead of the more expensive gas boiler. We can also notice the performance improvement when a capacity limit is imposed in the aggregation (e.g. $150 \mathrm{~kW}$ ), instead of the individual buildings (in that case $50 \mathrm{~kW}$ each). Therefore, the cost for the aggregator is smaller when the aggregated consumption is limited, because it can utilize its capacity among the buildings more economically. The cost difference for the unconstrained operation (total $P_{\text {cap }}>210 \mathrm{~kW}$ ) corresponds to the smaller imbalance costs achieved

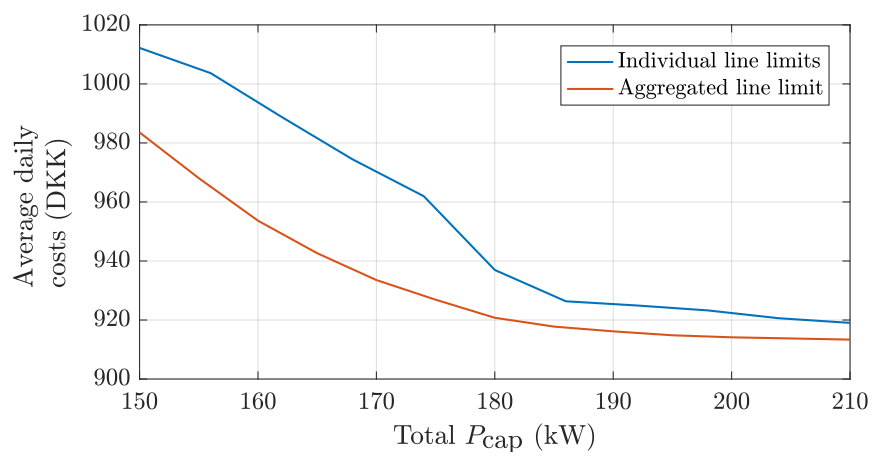

Fig. 4: Average daily costs for 3 buildings with individual line limits (blue colour) and an aggregated line limit (orange colour).

\section{DISCUSSION AND CONCLUSION}

We have presented a method for assessing the cost of imposing a capacity limit in the operation of the electrified heating system of individual buildings, as well as aggregations of buildings. We formulated the cost minimization problem as a MISP considering the imbalance costs and evaluated the cost of imposing capacity limits. We showed that the cost of such operational constraints can be quantified and the aggregator can use such a method to decide upon its optimal capacity limitation contract with the DSO or potentially sell such capacity products, which are very likely to appear in the future in the light of the increasing penetration and controllability of DERs. Moreover, we showed that aggregated capacity limits result in reduced costs; it would be therefore more economical for both an aggregator and DSO to trade such products, if the potential 
operational goals (voltage limits and grid congestions) allow it. Of course, in this case the location of the buildings (or DERs) is crucial, so that they can be aggregated under a common connection point, e.g. a feeder or a bus.

In our future work we will use more accurate models to represent buildings' dynamics, the various uncertainties in the heat demand, DA prices and load variability, as well as their potential interactions. We will also focus on the intra-quarterly fast load variations which we expect to have a significant impact on the individual line limits, but will increase the value of aggregation, due to the load-smoothing effects. We believe that capacity products are a relatively simple, reliable and easy to implement measure for the DSOs to operate their grids within secure limits, when the share of controllable loads with large power capacities, such as EVs, increases. It is therefore useful to investigate methods of evaluating the cost of imposing capacity limitations.

\section{REFERENCES}

[1] K. Clement-Nyns, E. Haesen, and J. Driesen, "The impact of charging plug-in hybrid electric vehicles on a residential distribution grid," IEEE Transactions on Power Systems, vol. 25, no. 1, pp. 371-380, Feb 2010.

[2] R. de Sá Ferreira, L. A. Barroso, P. R. Lino, M. M. Carvalho, and P. Valenzuela, "Time-of-use tariff design under uncertainty in priceelasticities of electricity demand: A stochastic optimization approach,' IEEE Transactions on Smart Grid, vol. 4, no. 4, pp. 2285-2295, Dec 2013.

[3] S. Huang, Q. Wu, S. S. Oren, R. Li, and Z. Liu, "Distribution locational marginal pricing through quadratic programming for congestion management in distribution networks," IEEE Transactions on Power Systems, vol. 30, no. 4, pp. 2170-2178, July 2015.

[4] B. Sivaneasan, K. N. Kumar, K. T. Tan, and P. L. So, "Preemptive demand response management for buildings," IEEE Transactions on Sustainable Energy, vol. 6, no. 2, pp. 346-356, April 2015.

[5] K. Margellos and S. Oren, "Capacity controlled demand side management: A stochastic pricing analysis," IEEE Transactions on Power Systems, vol. 31, no. 1, pp. 706-717, Jan 2016.

[6] J. Zou, S. Ahmed, X. A. Sun, and H. M. Stewart, "Nested Decomposition of Multistage Stochastic Integer Programs with Binary State Variables," Optimization Online, pp. 1-32, 2016.

[7] G. C. Pflug and A. Pichler, Multistage Stochastic Optimization. Springer International Publishing, 2014.

[8] B. Defourny, D. Ernst, and L. Wehenkel, "Multistage stochastic programming: A scenario tree based approach to planning under uncertainty," Decision Theory Models for Applications in Artificial Intelligence: Concepts and Solutions, pp. 97-143, 2012.

[9] Wamak website. [Online]. Available: http://www.wamak.eu/wapps/ datasheets/datasheet_v2.php?code=WA001047\&lang=en-GB

[10] Energinet.dk. [Online]. Available: http://www.energinet.dk/Electricity/ Energy-data

[11] J. Löfberg, "Yalmip : A toolbox for modeling and optimization in matlab," in In Proceedings of the CACSD Conference, Taipei, Taiwan, 2004.

[12] I. Gurobi Optimization, "Gurobi optimizer reference manual," 2016. [Online]. Available: http://www.gurobi.com 